\title{
Bedürfnistheorie des Menschen als Grundlage der Motivation der Arbeitsproduktivität
}

\author{
Boris M. Genkin
}

\section{Die Struktur des Menschenmodells in wirtschaftlichen Systemen}

Das wichtigste Hilfsmittel im Personalmanagement ist das Menschenmodell. In der Fachliteratur sind verschiedenste Menschenmodelle dargestellt worden. In der Regel gründen sie auf dem klassischen, in der ökonomischen Theorie und Praxis vorherrschenden Modell des ,homo oeconomicus“ [1], zum Beispiel das RREEMM-Modell (Resoursful, Restricted, Expecting, Evaluating, Maximizing Man) (Mecking, 1976). In der Soziologie wird das Modell des „homo soziologicus“, in der Psychologie des „homo psychologicus“" usw. benutzt.

Es ist anzunehmen, dass man bei der Anwendung des Menschenmodells im Personalmanagement von dem Komplexmodell, das ökonomische, soziale, ethische und psychologische Aspekte berüicksichtigt, ausgeht. In diesem Modell werden die vier Bereiche

1) Werte und Ziele,

2) Beduirfnisse,

3) Potenzial und

4) Motive unterschieden. Wechselbeziehungen zwischen diesen Bereichen sind in Abbildung 1 vorgestellt.

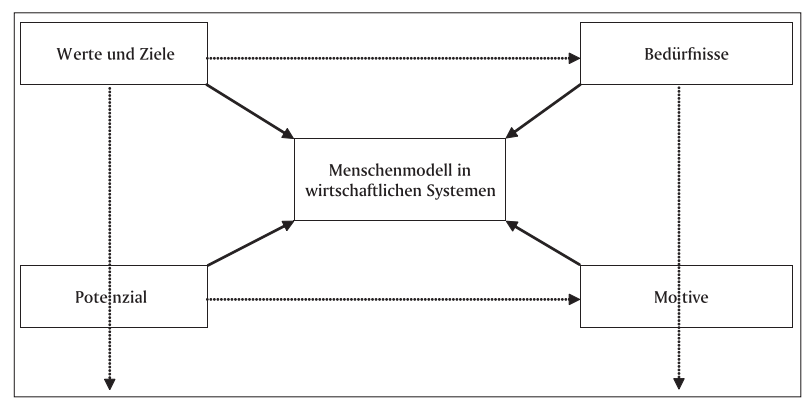

Abb.1: Struktur des Menschenmodells in wirtschaftlichen Systemen (Genkin, 2002: 40)

\section{Struktur des Motivationssystems}

Für die Organisation effektiver Arbeit ist es von großer Bedeutung, zwischen interner und externer Motivation zu unterscheiden. „Interne Motivation“ wird durch Inhalt und Bedeutung der ausgefuihrten Arbeit bestimmt. Ist sie interessant, dann wird sie von selbst zur Motivation einer aktiven und produktiven Tätigkeit. Neben dem Inhalt können auch die Bedeutung der Arbeit für die Entwicklung bestimmter Fähigkeiten einer Person, der Nutzen dieser Arbeit für andere Personen und für die Gesellschaft sowie die Übereinstimmung der Tätigkeit mit der Denkweise und ethischen Orientierung der Person eine wichtige Rolle bei der internen Motivation spielen. „Externe Motivation“ kann in drei Formen auftreten: administrativ, ökonomisch und statussymbolisch. „Administrative Motivation" bedeutet Ausfuihrung einer Arbeit auf Befehl, d. h. unter direktem Zwang in Verbindung mit der entsprechenden Bestrafung im Fall eines Verstoßes gegen bestimmte Normen. „Ökonomische Motivation“ funktioniert durch ökonomische Motive (Entgelt, Dividenden usw.). „Statussymbolische Motivation“ hat als Grundlage den Status des Mitarbeiters in der Organisation.

Die Effizienz der Motivation lässt sich durch das Ergebnis der Tätigkeiten einer Person bzw. einer Organisation sowie durch die Faktoren der Einstellung zur Arbeit (Anstrengung/Mühe, Hartnäckigkeit, Verantwortungsbewusstsein, Aufmerksamkeit, Verhalten) beurteilen. Abbildung 2 zeigt die Interdependenz zwischen verschiedenen Elementen des Motivationsprozesses. Das Verhalten einer Person kann durch Befehle, ökonomische Stimuli sowie durch Werteinstellungen, Interessen und Beduirfnisse beeinflusst werden.

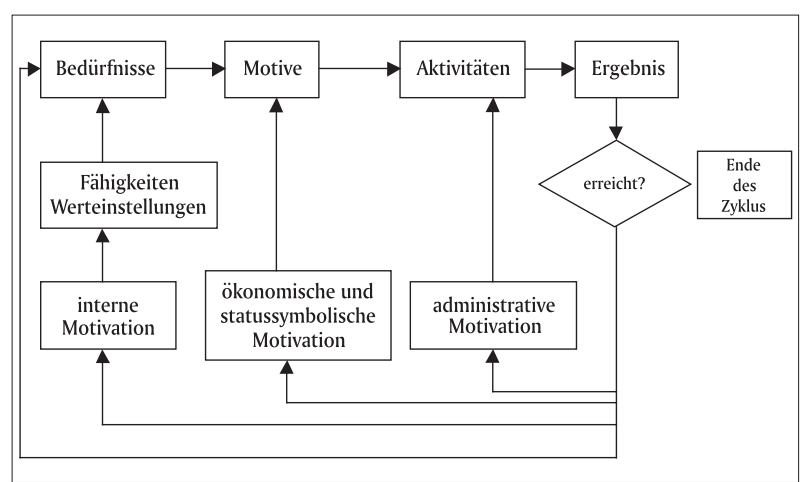

Abb. 2: Interdependenzen in Rahmen des Motivationsprozesses (Genkin 2003)

\section{Prinzipien der allgemeinen Bedürfnis- theorie: Wechelbeziehungen der ökonomischen, psychologischen und synergetischen Aspekte}

Zurzeit entwerfen und verwenden zwei wissenschaftliche Disziplinen eigene Bedürfnistheorien - die Psychologie und die Ökonomie. Diese Theorien existieren praktisch unabhängig voneinander. Ökonomische Theorien, die auf den Ideen des marginalen Nutzens gegründet sind, finden ihre Anwendung im Bereich der Mikroökonomie. Psychologische Theorien (von Maslow, Herzberg, Alderfer u. a.) werden üblicherweise im Personalmanagement benutzt und in den entsprechenden Lehrbüchern erörtert (z. B. Scholz u. a.). 
Bei der Beurteilung der Qualität dieser Bedürfnistheorien soll Folgendes berücksichtigt werden:

1) der ganze Diapason der Bedürfnisse (Forderung der Ganzheit);

2) die individuellen Besonderheiten der Menschen, ihrer Ziele und Werte (Forderung der Ziel- und Wertindividualisierung);

3) die Prioritäten und Niveaus der Befriedigung von Bedürfnissen (Forderung der Hierarchisierung);

4) die Dynamik der Bedürfnisformation, die den Mechanismus ihrer Wechselbeziehungen bestimmt (Forderung der Dynamik).

Die bekannten Beduirfnistheorien genügen diesen Forderungen nicht. Eine solide Analyse bot die Maslowsche Theorie, die 1943 veröffentlicht wurde. Zu den Vorzügen von Maslow im Vergleich mit anderen gehören:

- die Berechnung der individuellen Bedürfnisse des Menschen (besonders einer schaffenden Persönlichkeit) sowie

- einige Vorschläge für die Dynamik der Bedürfnisse.

Bei der Darlegung der Maslowschen Theorie in verschiedenen Lehrbüchern für Management, Marketing und Soziologie steht die so genannte Bedürfnispyramide im Mittelpunkt, was sehr verwunderlich ist, da Maslow in seinen eigenen Büchern und Artikeln zwar Bedürfnisse klassifizierte, zugleich aber auch die Relativität der Bedürfnishierarchie unterstrich sowie die Individualität und die Ziele der Menschen berücksichtigte. Folglich stellte er in keiner seiner Arbeiten eine allgemeinguiltige Beduirfnispyramide auf oder verwendete ihr ähnliche Abbildungen. Vielmehr lässt sich aus den Texten des Gründers der humanistischen Psychologie herauslesen, dass eine Bedürfnishierarchie eben gerade nicht starr und allgemeingültig sein kann, denn Maslow betont die besondere Wichtigkeit der Strukturindividualisierung von Bedürfnissen für die Menschen, die nach einer kreativen Tätigkeit streben.

Wenn Maslow keine Pyramide erstellen konnte, dann fragt sich, warum diese in den Lehrbüchern dutzender Autoren in verschiedenen Ländern erscheint. Es ist sehr wahrscheinlich, dass die Antwort auf diese Frage der bekannten Anekdote darüber, dass die Menschen gewöhnlich nicht dort suchen, wo sie etwas verloren haben, sondern dort, wo es heller ist, ähnelt. Offensichtlich entschied einer der Autoren, dass es mit einer Pyramide leichter ist, den Studenten und Schülern die Idee der Bedürfnishierarchie zu erklären. In der deutschsprachigen Literatur kann man die Bedürfnispyramide zumindest ab 1975 in der ersten Auflage des Lehrbuches von U. Stopp (S. 141) finden. In diesem Buch ist Maslow jedoch nicht als Urheber der entsprechenden Abbildung angegeben. Möglicherweise gibt es auch frühere Publikationen zu diesem Thema.

Obwohl ohne Zweifel eine gewisse Reihenfolge in der Bedürfnisbefriedigung existiert, trifft diese nicht auf alle Menschen gleichermaßen zu. Die Lebensläufe bedeutender Wissenschaftler, Künstler und Theologen zeigen, dass Bedürfnisse nach Kreativität und geistiger
Vervollkommnung nicht erst nach der Befriedigung aller übrigen Bedürfnisse (physiologische, soziale Zugehörigkeit, Anerkennung usw.) entstehen, sondern im Grunde genommen schon an der Überlebensgrenze, wenn die Grundbeduirfnisse nach Nahrung und Sicherheit noch nicht befriedigt sind, dominieren können.

Die Erfahrung zeigt, dass die Bedürfnishierarchie vornehmlich personenabhängig ist. Als eine Gemeinsamkeit kann man nur die Tatsache gelten lassen, dass eine Befriedigung von Grundbedürfnissen keine notwendige Bedingung für die Entwicklung von anderen höheren Bedürfnissen ist. Daraus folgt, dass man bei der Beduirfnisklassifikation nicht nur ihre Arten, sondern auch das Niveau der Befriedigung berüicksichtigen muss. Folgende Faktoren werden in allen uns bekannten Klassifikationsschemata nicht berücksichtigt:

- die individuellen Unterschiede nach Zusammensetzung, Hierarchie und Wichtigkeit von Bedürfnissen,

- das Niveau der Bedürfnisbefriedigung,

- die Abhängigkeit der Bedürfnisse von Wertschätzungen und Lebenszielen des Menschen.

\section{Niveaus der Bedürfnisbefriedigung und der Lebensziele der Menschen}

Für die Berüicksichtigung dieser Faktoren ist es sinnvoll, zuerst alle vorhandenen Bedürfnisse in zwei Gruppen aufzuteilen, und zwar in

- Existenzbedürfnisse und

- Bedürfnisse nach Erreichen von Lebenszielen.

$\mathrm{Zu}$ der ersten Gruppe gehören üblicherweise die Bedürfnisse nach Nahrung, Kleidung und Sicherheit. Dazu sollte man auch das Bedürfnis nach sozialer Zugehörigkeit zählen. Es wird dadurch bestimmt, dass ein Mensch uiber längere Zeit außerhalb einer sozialen Gruppe nicht existieren kann.

Man kann zwei Grundniveaus der Existenzbedürfnisse bestimmen:

- das Mindestniveau und

- das Basisniveau.

Das „Mindestniveau“ sichert das physische Überleben des Menschen. Das „Basisniveau“ ermöglicht eine Entwicklung von bedeutenden intellektuellen und psychischen Bedürfnissen. Das Niveau kann sowohl subjektiv als auch objektiv festgestellt werden. Im ersten Fall kann als Kriterium für das Erreichen des Basisniveaus die Zeit dienen, die man zum Nachdenken über eine Befriedigung der Essens-, Wohnungs- und Sicherheitsbeduirfnisse braucht. Man kann annehmen, dass man dafür die Hälfte der gesamten Tageszeit brauchen würde. Objektiv lässt sich das Basisniveau festmachen am Konsumbudget, das von Experten für die unterschiedlichen Tätigkeiten als notwendig eingeschätzt wird.

Auf den Basisniveaus der Befriedigung der Existenzbedürfnisse entwickeln sich die Bedürfnisse nach Erreichen von Lebenszielen, die in fünf Gruppen unterteilt sind: 
1) materielle Beduirfnisse,

2) soziale Bedürfnisse,

3) intellektuelle Bedürfnisse,

4) ästhetische Beduirfnisse und

5) geistige Beduirfnisse.

Die „materiellen Bedürfnisse“, die in einer entsprechenden sozialen Gruppe das Basisniveau überschreiten, kann man als Luxusbedürfnisse betrachten. Man muss berüicksichtigen, dass der Luxusbegriff in bestimmten Massen relativ ist: Das, was für eine soziale Gruppe Luxus bedeutet, ist für eine andere Norm (d. h. was dem Basisniveau der Existenzbeduirfnisse entspricht). Allgemein bekannt ist das so genannte „demonstrative Konsumverhalten“, das den Wunsch nach Zugehörigkeit zu einer „höheren“ sozialen Gruppe unterstreicht. Eine Charakteristik dieses Verhaltens gibt es in Arbeiten von z. B. A. Marschall und vielen anderen Autoren.

„Soziale Bedürfnisse“ kann man in egoistische und altruistische Bedürfnisse aufteilen. Zu den ersten gehören Freiheits-, Macht-, Ruhm-, Anerkennungs- und Achtungsbedürfnisse. Zu den zweiten gehören Bedürfnisse nach Wohltätigkeit, Liebe zu Kindern, Eltern und anderen Menschen.

Unter ,intellektuellen Bedürfnissen“ versteht man das Streben nach Wissen und in der Wissenschaft nach Kreativität.

Die „ästhetischen Bedürfnisse“ sind durch das Streben nach Schönheit und in der Kunst nach Kreativität bedingt.

Zu den „geistigen Bedürfnissen“ gehören das Streben nach einer geistigen Perfektion, einem Glauben, der Liebe zu Gott und Wahrheit.

In Abhängigkeit von den individuellen Neigungen, Fähigkeiten und Ansprüichen wird bei den einen Menschen nach Erreichen der Basisebene der Existenzbedürfnisbefriedigung das Streben nach einer Maximierung des Verbrauchs an materiellen Gütern dominieren, bei den anderen das Streben nach Macht und Ruhm, bei dritten nach Wissen und Kreativität, bei vierten nach Kunst und bei den fünften nach geistiger Vervollkommnung.

Die Struktur der Bedürfnisse kann sich bei einem und demselben Menschen im Verlauf seines Lebens mehrmals ändern. Je niedriger dabei das subjektiv als normal eingeschätzte Basisniveau der Bedürfnisse ist, desto wahrscheinlicher ist es, dass nach dem Erreichen des Niveaus die intellektuellen und geistigen Bedürfnisse dominieren werden.

Die Bedürfnisse nach Erreichen von Lebenszielen können charakterisiert werden als

- das Streben nach größerer Existenzbeduirfnisbefriedigung (dies bezieht sich auf das Bedürfnis nach Luxus, Anerkennung, Ruhm),

- die Entstehung neuer Bedürfnisgruppen (wie Wissen, Kreativität und geistige Vervollkommnung).
Zwischen den fünf aufgelisteten Bedürfnisgruppen gibt es keine genau festgelegten Grenzen. Insbesondere kann das Streben nach Luxus in vielen durch Eitelkeit hervorgerufen werden und ein Streben nach Macht ist nicht selten ein Mittel des Zugangs zum Luxus. Dabei dominieren die Bedürfnisse der einen Gruppe im Verlauf eines bestimmten Zeitraumes.

Insgesamt kann man die Bedürfnisstruktur eines Menschen wie in Abbildung 3 darstellen. Der Unterschied zwischen den Basisniveaus der Befriedigung von Existenzbedürfnissen für verschiedene Menschen und Tätigkeitsarten ist darin durch eine stufenförmige Linie dargestellt.

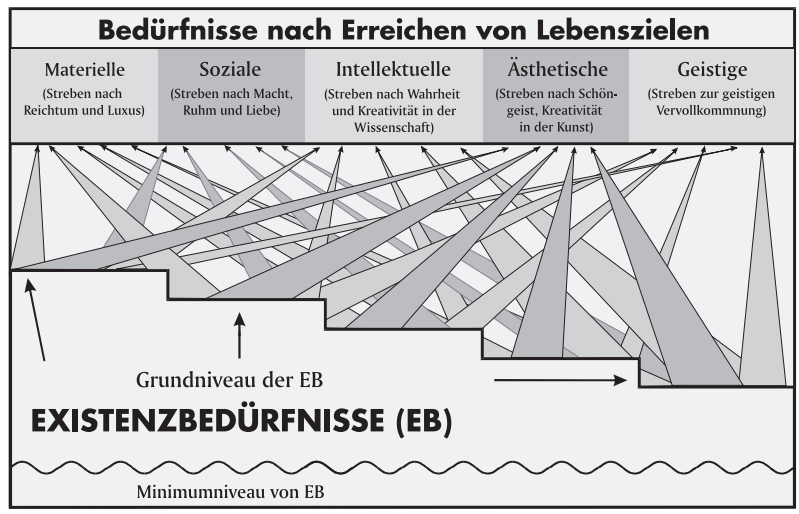

Abb. 3. Das Modell der Menschenbedürfnisstruktur (Genkin, 2003: 70)

Wie aus Abbildung 3 ersichtlich, gehören die Bedürfnisse zum Erreichen von Lebenszielen zu einem Hierarchieniveau, d. h. es gibt keinen Grund für die Annahme, dass die sozialen Bedürfnisse den intellektuellen und geistigen vorangehen müssen oder umgekehrt.

\section{Die Bedürfnisdynamik: Synergetische und mikroökonomische Betrachtungs- weisen}

Den Entwicklungsprozess von intellektuellen und psychischen Bedürfnissen kann man mit der synergetischen Terminologie als Prozess des Übergangs vom Zustand des „Chaos“, dem die menschliche Tätigkeit der Befriedigung von Existenzbedürfnissen entspricht, zum Zustand des „Kosmos“ beschreiben, in dem die Bedürfnisse nach Erreichen von Lebenszielen dominieren. Dieser Prozess vollzieht sich unter dem Einfluss von Interessen, Werten, Inspirationen usw., die durch die Fähigkeiten eines Menschen und ihre Realisierungsbedingungen bedingt sind.

Für eine Quantitätsanalyse der Bedürfnisdynamik kann die mikroökonomische Konsumtheorie angewendet werden, die eine Abhängigkeit zwischen dem Bedürfnisbefriedigungsgrad, den Konsumentenressourcen (Geld, Zeit) und den Preisen der Konsumguiter annimmt.

Das erste Gossensche Gesetz besagt, dass der Grad der Befriedigung (subjektiver Nutzen) bei der ersten konsumierten Einheit am größten ist und bei jeder weiteren beständig abnimmt. Als empirische Grundlage dessen 
gilt das psycho-physiologische Gesetz von Weber-Fechner, nach dem sich wiederholende Reize der gleichen Intensität zu einer Minderung der Empfindungsintensität führen.

Für Individuen mit Kreativitätsneigungen sind der Schöpfungsprozess und dessen Ergebnisse am erstrebenswertesten, dessen subjektiver Nutzen sich nach seinem Konsum (im Rahmen der physischen Möglichkeiten) nicht verringert. Im Unterschied zum Konsum materieller Güiter ordnen sich die schöpferischen Tätigkeiten den Gesetzen von Gossen und Weber-Fechner nicht unter.

Die Bedürfnisse nach geistiger Vervollkommnung, Liebe (zu Gott, Kindern und Freunden), Ruhm, Macht und die meisten anderen sozialen Beduirfnisse bleiben von oben genannten Gesetzen unabhängig. Die Reichtums- und Luxusbedürfnisbefriedigung verhalten sich der Machtbedürfnisbefriedigung identisch: Menschen, die entsprechend orientiert sind, streben in der Regel immer mehr nach Reichtum und Luxus. Somit unterscheiden sich Bedürfnisse nach Erreichen von Lebenszielen von Existenzbedürfnissen nicht nur inhaltlich, sondern auch nach formellen Merkmalen: Die Gesetze von Gossen und Weber-Fechner gelten nur für Existenzbedürfnisse.

Innerhalb der Existenzbedürfnisse besteht im Allgemeinen für alle Menschen die traditionelle Hierarchie von Beduirfnissen (physiologische - Sicherheit - Beziehung) nur im Rahmen des minimalen Befriedigungsniveaus, d. h. an der Überlebensgrenze. Oberhalb dieses Niveaus bildet sich eine Reihenfolge der Befriedigung von Existenzbedürfnissen gemäß der jeweiligen Individualität des Menschen und der konkreten Situation. Die Existenzbedürfnisse von Menschen, die auf Wissen, Schaffen und geistige Vervollkommnung orientiert sind, sind in der Regel niedriger als von denen, die Reichtum und Macht als die wichtigsten Werte empfinden.

Der Übergang von Existenzbedürfnissen zu Bedürfnissen nach Erreichen von Lebenszielen verwirklicht sich unter dem Einfluss der Attraktoren (Interessen, Werte, Begeisterung), die durch die Fähigkeiten der Menschen und die Bedingungen für ihre Realisierung hervorgerufen sind.

Die Dynamik der Bedürfnisse ist zweckmäßig in drei Perioden zu unterteilen, in die strategische, die taktische und die operative. Die Gesetze der Theorie der Grenznutzen treffen nur auf Existenzbedürfnisse zu. Bedürfnisse nach Erreichen von Lebenszielen können hingegen objektiv keine Grenzen der Befriedigung haben. Besonders betrifft das die Bedürfnisse nach Schaffen und geistiger Vervollkommnung.

Der vorliegende Ansatz unterscheidet von anderen Ansätzen hauptsächlich wie folgt:

1) Die Bedürfnisse werden in zwei Kategorien unterteilt, in Existenzbedürfnisse und in Bedürfnisse nach Erreichen von Lebenszielen.

2) Es gibt zwei Ebenen der Befriedigung von Existenzbedürfnissen, das Mindest- und das Basisniveau.
3) Die Basisebenen der Befriedigung von Existenzbedürfnissen können beträchtliche individuelle und Gruppenunterschiede haben.

4) Nach dem Basisniveau der Befriedigung der Existenzbedürfnisse dominieren die Bedürfnisse nach Erreichen von Lebenszielen.

5) Anhand einer synergetischen und mikroökonomischen Analyse wurde eine Dynamik der Bedürfnisformierung erforscht.

\section{Praktische Aspekte}

Die Verbesserung der Motivationssysteme im Betrieb beginnt zweckmäßig mit der Analyse der Meinungen der Mitarbeiter. Diese zu analysierenden Meinungen zeigt uns der nachfolgende Fragebogen.

\begin{tabular}{|c|c|}
\hline \multicolumn{2}{|l|}{ Sehr geehrter Kollege! } \\
\hline \multicolumn{2}{|c|}{$\begin{array}{l}\text { In unserem Betrieb wollen wir die Arbeitsorganisation, Arbeitsnormung } \\
\text { und Arbeitsentlohnung verbessern. Deshalb bitten wir Sie, folgende } \\
\text { Fragen zu beantworten: }\end{array}$} \\
\hline \multicolumn{2}{|l|}{$\begin{array}{l}\text { 1. Schätzen Sie bitte Ihre Zufriendenheit mit der Arbeit in } \\
\text { den folgenden Punkten ein (in Prozenten): }\end{array}$} \\
\hline Niveau des Arbeitslohnes & \\
\hline $\begin{array}{l}\text { Bereitstellung von Dienstleistungen des Betriebes } \\
\text { für die Mitarbeiter }\end{array}$ & \\
\hline Gerechtigkeit der Entlohnung & \\
\hline Arbeitsbedingungen am Arbeitsplatz & \\
\hline $\begin{array}{l}\text { Möglichkeit, im Beruf vowärts zu kommen und } \\
\text { Karriere zu machen }\end{array}$ & \\
\hline $\begin{array}{l}\text { Möglichkeit der Ausbildung im Betrieb, in den } \\
\text { Hochschulen und in anderen Lehranstalten }\end{array}$ & \\
\hline $\begin{array}{l}\text { Verhältnis Ihrer Arbeitskollegen untereinander } \\
\text { (Team, Abteilung, Gruppe) }\end{array}$ & \\
\hline Ihr Verhältnis zu Ihrem Vorgesetzten & \\
\hline Möglichkeit der frei einteilbaren Arbeitszeit & \\
\hline $\begin{array}{l}\text { 2. Wie groß ist insgesamt der Anteil der kreativen } \\
\text { Tätigkeiten an Ihrer Arbeit? }\end{array}$ & \\
\hline \multicolumn{2}{|l|}{ 3. Wieviel Prozent der Mitarbeiter rufen bei Ihnen hervor ... } \\
\hline ... positive Emotionen & \\
\hline ... negative Emotionen & \\
\hline
\end{tabular}

Tabelle 1: Fragebogen

Grundlage der wirtschaftlichen Motivation ist die Gegenüberstellung der normativen (planmäßigen) und der wirklichen Kosten der Ressourcen. Die optimalen Normen der Ressourcenkosten sollen dem Minimum der summarischen Ressourcenkosten bezogen auf den Produktionsausstoß, der durch die Schaffung von ergonomischen Arbeitsbedingungen erreicht wird, entsprechen. Die Methodik der entsprechenden Berechnungen ist im Lehrbuch (Genkin, 2004) vorgestellt. Die Zunahme des Arbeitslohnes soll der Senkung der Normen der Ressourcenkosten je Produktionseinheit entsprechen. Die Dynamik der Kennziffern, die in Abbildung 4 vorgestellt ist, soll eine Grundlage der Tarifverhandlungen sein. 


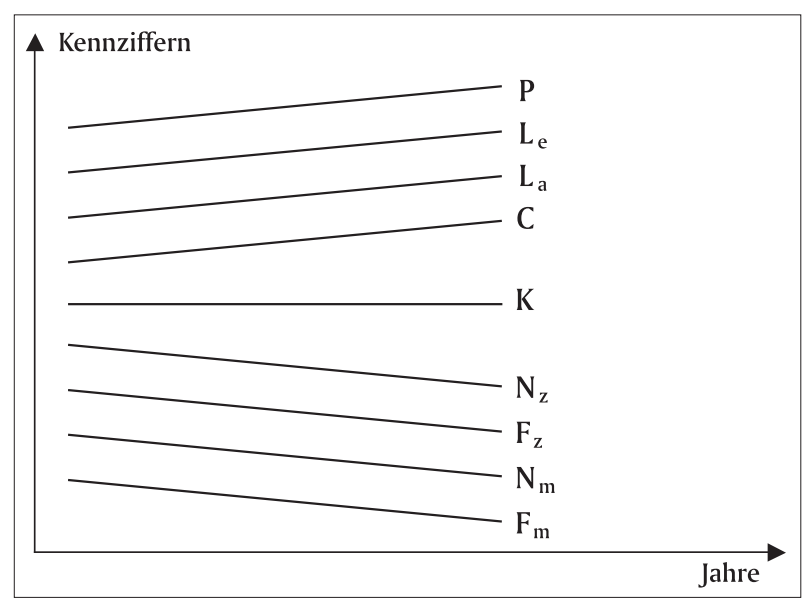

Abb. 4: Die Dynamik der wirtschaftlichen Kennziffern bei der rationalen Unternehmensorganisation (P - Produktivität; La-Arbeitslohn der Arbeiter; Le - Arbeitslohn der Ingenieure; C - Tarifsätze, Zuschläge (Zulagen), Prämie; $\mathrm{K}$ - Koeffizient der Normerfüllung; $\mathrm{Nz}$ - Zeitnormen; $\mathrm{Fz}$ - wirkliche Kosten der Zeit; Nm - die Normen der Kosten materieller Ressourcen; Fm - wirkliche Kosten materieller Ressourcen bezogen auf die Produktionseinheit.

\section{Anmerkungen}

[1] „Homo oeconimicus“- Der so umschriebene Mensch hat die eigene Nutzenmaximierung als Ziel gesteckt, er verfolgt Ziele einzig nach dem Eigeninteresse. Da sich aber das Verfolgen seines Interesses mit dem Interesse einer größeren Gruppe deckt bzw. Folgen nach sich zieht, die dem Gemeinwohl dienen, ist dieser eher egozentrisch anmutende Begriff allgemeinverträglich. (amazon.de an der uni, 1999)

\section{Literatur}

Genkin, B. M.: Ekonomika i soziologia truda. (Arbeitsökonomie und -soziologie), 5. erweiterte Auflage, Moskau: Norma, 2003 (russisch).

Genkin, B. M.: Wwedenie w metaekonomiku i osnowanija ekonomitscheskich nauk. (Einfuihrung in die Metaökonomik und Grundlagen der Wirtschaftswissenschaften), Moskau: Norma 2002 (russisch).

Genkin, B. M.: Organizazija truda (Arbeitsorganisation), 2. Auflage. Moskau: Norma 2004 (russisch).

Maslow, A.: Theory of Human Motivation. Psychological Review 50, 1943.

Mecking, W. H.: Values and the Choice of the Model of the Individual in the Social Sciences, Schweizerische Zeitschrift fuir Volkswirtschaft und Statistik, Bd. 112, 1976, S. 545-559.

Scholz, C.: Personalmanagement. München: F. Vahlen, 1993.

Stopp, U.: Betriebliche Personalwirtschaft. 1. Auflage, Stuttgart: Springer Verlag, 1975.

\section{Autor}

\section{Prof. Dr. Boris M. Genkin}

Staatliche Universität für Ingenieur- und Wirtschaftswissenschaften St. Petersburg

27, Marata St., 191002 St. Petersburg, Russland

Tel. +7 8121120604

E-Mail: pers@engec.ru 